\section{Reaction of hexatantalate species with Layered Double Hydroxide}

\author{
RANA CHOUMANE ${ }^{1}$, VICTOR CARPENTIER ${ }^{2}$ AND \\ GRÉGORY LEFÈVRE ${ }^{1}$
}

${ }^{1}$ PSL Université, Chimie ParisTech - CNRS, Institut de Recherche de Chimie Paris

${ }^{2}$ TND

Presenting Author: rana.choumane@chimieparistech.psl.eu

Tantalum has become a key element in our society, due to its importance in electronics. Its behavior in environment should be better understood, in particular to address its potential impact. Thus, its interactions with solids, naturally occurring or designed for its uptake are worth to be studied.

$\mathrm{Ta}(\mathrm{V})$ is soluble as hexatantale species $\left(\mathrm{H}_{\mathrm{x}} \mathrm{Ta}_{6} \mathrm{O}_{19}{ }^{(6-\mathrm{x})-}\right)$ in alkaline solutions, which has a polyoxometalate (POM) geometry. A strong interaction between POM and layered double hydroxide (LDH) has been reported for $\mathrm{W}$ and Mo due to the very high negative charge of the POM, leading to their insertion between the positively charged sheets. Other reactions were nevertheless observed, such as their adsorption on the surface of the particles. So far, no studies have been performed with Ta.

We have first quantified the sorption of hexatantale species on a LDH (Mg-Fe ${ }^{\mathrm{III}}$-carbonate), and the sorbed amount corresponded to only $14 \%$ of the anionic exchange capacity (AEC). XRD characterization of LDH before and after reaction with Ta has shown no change in its diffractogram (Figure 1), what leads to the counter-intuitive result that hexatantale is not intercalated in the LDH structure. Despite the high sensitivity of tantalum in XRD, no new peaks are present in the diffractogram, leading to the hypothesis of an adsorption. This interpretation has been confirmed by zeta potential measurements. A second series of sorption experiments has been performed using the LDH after calcination at $400^{\circ} \mathrm{C}$, following a usual protocol of activation of this solid to enable its reconstruction by "memory effect". This results in a sorption of Ta equivalent to $100 \%$ of the AEC. Nevertheless, the diffractogram of this sample does not correspond to the structure of a $\mathrm{LDH}$, and consists in a mixture of metal oxides $\left(\mathrm{MgO}\right.$ and $\mathrm{MgFe}_{2} \mathrm{O}_{4}$ ) with additional peaks not identified in commercial XRD databases. The precipitation of a $\mathrm{Mg}-\mathrm{Ta}(\mathrm{V})$ phase has been assumed, and was confirmed by $\mu$ Raman analyses and dedicated experiments based on the reaction of hexatatantalate solutions with $\mathrm{MgO}$ or $\mathrm{MgCl}_{2}$ solution.

Thus, two different reactions between $\mathrm{Ta}(\mathrm{V})$ and a carbonated LDH Mg-Fe have been identified, along with a new $\mathrm{Mg}-\mathrm{Ta}(\mathrm{V})$ solid phase.

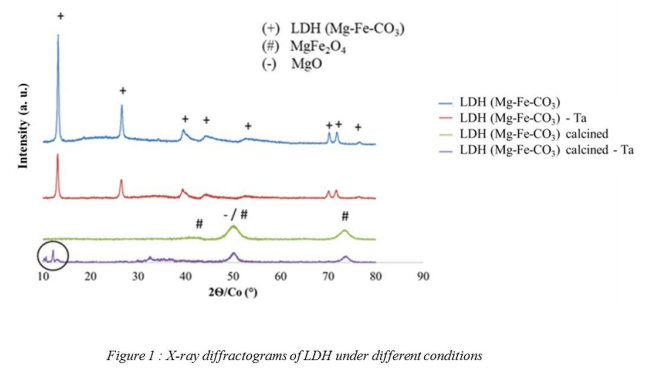

